\title{
Suppression of PPAR $\gamma$ through MKRN1-mediated ubiquitination and degradation prevents adipocyte differentiation
}

\author{
J-H Kim ${ }^{1,4}$, KW Park ${ }^{2,4}$, E-W Lee ${ }^{1}$, W-S Jang ${ }^{2}$, J Seo ${ }^{1}$, S Shin ${ }^{1}$, K-A Hwang ${ }^{3}$ and J Song ${ }^{\star, 1}$
}

The central regulator of adipogenesis, PPAR $\gamma$, is a nuclear receptor that is linked to obesity and metabolic diseases. Here we report that MKRN1 is an E3 ligase of PPAR $\gamma$ that induces its ubiquitination, followed by proteasome-dependent degradation. Furthermore, we identified two lysine sites at 184 and 185 that appear to be targeted for ubiquitination by MKRN1. Stable overexpression of MKRN1 reduced PPAR $\gamma$ protein levels and suppressed adipocyte differentiation in 3T3-L1 and C3H10T1/2 cells. In contrast, MKRN1 depletion stimulated adipocyte differentiation in these cells. Finally, MKRN1 knockout MEFs showed an increased capacity for adipocyte differentiation compared with wild-type MEFs, with a concomitant increase of PPAR $\gamma$ and adipogenic markers. Together, these data indicate that MKRN1 is an elusive PPAR $\gamma$ E3 ligase that targets PPAR $\gamma$ for proteasomal degradation by ubiquitin-dependent pathways, and further depict MKRN1 as a novel target for diseases involving PPAR $\gamma$.

Cell Death and Differentiation (2014) 21, 594-603; doi:10.1038/cdd.2013.181; published online 13 December 2013

Excessive body fat in obese individuals is considered a major cause of insulin resistance, cardiovascular diseases, and diabetes. ${ }^{1}$ In obesity, adipocytes alter the status of energy homeostasis by the generation of adipokines, demonstrating that adipocytes have active roles in energy homeostasis and body composition, in addition to storage of triglycerides. ${ }^{2}$ Adipokines secreted by obese adipocytes such as resistin and TNF- $\alpha$ impair insulin actions in peripheral tissues, whereas adiponectin from lean adipose tissue improves insulin sensitivity in liver and muscle. ${ }^{2,3}$ Therefore, a better understanding of adipocyte biology and mechanisms that direct adipocyte differentiation is being rapidly sought. ${ }^{4}$

Adipocyte differentiation from pre-adipocytes is controlled by a number of transcriptional cascades, including peroxisome-proliferator-activated receptor $\gamma$ (PPAR $\gamma$ ), the CCAAT/ enhancer-binding protein (C/EBP) family, and adipocyte determination and differentiation-dependent factor. ${ }^{5}$ Lossand gain-of-function studies have demonstrated that PPAR $\gamma$ is a necessary and sufficient transcriptional regulator to convert adipocytes from their precursors. ${ }^{6,7}$ Activation of PPAR $\gamma$ by its ligands induces PPAR $\gamma$ binding to its responsive elements in the promoter, and increases the expressions of lipid synthesis- and differentiation-related genes, including
$C D 36$, lipoprotein lipase $(L P L)$, fatty acid-binding protein $(a P 2)$, and $C / E B P \alpha^{8}{ }^{8}$ Upstream transcriptional events that induce the expression of PPAR $\gamma$ are C/EBP $\beta, \operatorname{C/EBP} \delta$, Krox20, KLF5, EBF, ZNF453, TCF7L2, and ADD1/SREBP1C transcription factors. ${ }^{4,5,9}$ In contrast, inhibitory transcriptional regulators such as KLF2, GATA2/3, IRF, and $\beta$-catenin/TCF have been shown to suppress the expression of PPAR $\gamma$ and

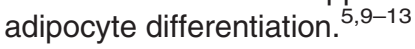

PPAR $\gamma$ protein is also post-translationally regulated by several modifications. ${ }^{14}$ Phosphorylation on serine 122 in the AF1 region of PPAR $\gamma$ is modified by ERK1/2 and P38/JNK kinases activated from growth factors, cytokines, and stress signals, and leads to inhibition of PPAR $\gamma$ activity and adipocyte differentiation. ${ }^{14,15}$ Sumoylation on lysine residue 395 targets PPAR $\gamma$ to NCoR corepressor and prevents repression of inflammatory genes. ${ }^{16}$ A short-lived PPAR $\gamma$ protein has been shown to be polyubiquitinated and degraded in a proteasome-dependent manner. ${ }^{17,18}$ Recent RNAi-based screening has shown that ubiquitin ligase Siah2 probably regulates PPAR $\gamma$ coregulator (NCoR, $\mathrm{RXR} \alpha$ ), thereby inhibiting PPAR activity in mature 3T3-L1 adipocytes. ${ }^{19}$ Another report also showed that ubiquitin ligase NEDD4-1 ubiquitinates PPAR $\gamma$ protein. $^{20}$ These data indicate that various

${ }^{1}$ Department of Biochemistry, College of Life Science and Biotechnology, Yonsei University, Seoul 120-749, Republic of Korea; ${ }^{2}$ Department of Food Science and Biotechnology, College of Biotechnology and Bioengineering, Sungkyunkwan University, Suwon, Gyeonggi-do 440-746, Republic of Korea and ${ }^{3}$ Department of Agrofood Resources, National Academy of Agricultural Science, RDA, Suwon 441-853, Republic of Korea

*Corresponding author: J Song, Department of Biochemistry, Yonsei University, Yonsei-ro 50, Seodaemun-gu, Seoul 120-749, Republic of Korea. Tel: + 8222123 5695; Fax: + 822362 9897; E-mail: jso678@yonsei.ac.kr

${ }^{4}$ These authors contributed equally to this work.

Keywords: PPAR $\gamma$; MKRN1; ubiquitination; adipocyte differentiation

Abbreviations: 3T3-L1, murine preadipocytic line derived from NIH-3T3; C3H10T1/2, Mesenchymal stem cell line, fibroblasts; H1299, human lung cancer cell line; PC-3, prostate cancer cell line; HEK293T, human kidney cancer cell line; MEFs, Mouse embryonic fibroblasts; ATCC, American Type Culture Collection; PPAR $\gamma$, peroxisome-proliferator-activated receptor $\gamma$; C/EBP, $\beta$ and $\delta$, CCAAT/enhancer-binding proteins, $\beta$ and $\delta$; LPL, lipoprotein lipase; aP2, fatty acid-binding protein; MKRN1, Makorin Ring Finger Protein 1; CD36, Cluster of Differentiation 36; NR, nuclear receptor superfamily; qRT-PCR, real-time reverse-transcription PCR; DMI, A cocktail of dexamethasone, 3-isobutyl-1-methylxanthine, and insulin; DI, A cocktail of dexamethasone and insulin, IBMX, 3-isobutyl-1-methylxanthine; DMSO, dimethyl sulfoxide; IP, immunoprecipitation; NP40, Nonidet P-40; PBS, phosphate-buffered saline; CHX, cycloheximide; Tro, Troglitazone; NEM, N-ethylmaleimide; pBP, pBABEpuro empty vector; shRNA, short-hairpin RNA; Ub, Ubiquitin; WCE, whole-cell extracts; WB, western blot

Received 14.5.13; revised 5.11.13; accepted 11.11.13; Edited by M Piacentini; published online 13.12.13 
post-translational modifications are integral players responsible for the physiological effects of PPAR $\gamma$. Therefore, the identification and elucidation of the mechanisms of posttranslational modification will help us to understand the physiological functions of PPAR $\gamma$, adipocyte biology, and further applications to therapeutic interventions.

Makorin Ring Finger Protein 1 (MKRN1) is known to act as an E3 ubiquitin ligase, targeting various substrates, such as hTERT, p53, and FADD. ${ }^{21-25}$ It may also function as a regulator of nuclear receptors, including androgen and retinoic acid receptors, independent of E3 ligase activities. ${ }^{26}$ In this study, we report that MKRN1 may mediate PPAR $\gamma 2$ ubiquitination and proteasome-dependent degradation. The physiological function of MKRN1 in negative regulation of PPAR 22 protein was further demonstrated using MKRN1 knockout MEFs, 3T3-L1, and C3H10T1/2 cell lines, indicating that MKRN1 is a potential new therapeutic target in PPAR $\gamma$ related diseases.

\section{Results}

PPAR 2 protein levels are regulated by MKRN1. In previous studies, MKRN1 was shown to repress the transcriptional activities of nuclear receptors such as androgen and retinoic acid receptors. ${ }^{26}$ This finding prompted us to investigate whether MKRN1 also has regulatory effects on the transcriptional activities of PPAR $\gamma$, a nuclear receptor responsible for adipocyte differentiation. ${ }^{27}$ The effects of MKRN1 on PPAR $\gamma 2$ were examined by co-transfecting H1299 human lung cancer cells with plasmids expressing PPAR $\gamma 2$ and PPRE-luciferase reporter in the presence or absence of troglitazone, an agonist of PPAR $\gamma 2$. The expression of PPAR $\gamma 2$ stimulated reporter gene expression, and this induction was further increased in the presence of troglitazone. Interestingly, MKRN1 co-expression with PPAR $\gamma 2$ was able to suppress the transcriptional activities to basal levels, implicating a negative regulatory role of MKRN1 on PPAR 2 activities (Supplementary Figure 1a). To analyze the effects of MKRN1 more critically, we used MKRN1 depleted in $\mathrm{H} 1299$ followed by luciferase reporter assays. Two independent MKRN1 siRNA \#5 and \#6 (siMK1\#5 and \#6, respectively) suppressed levels of endogenous MKRN1 up to 90\% in H1299 (Supplementary Figure 1b). The data indicated that, under depletion of MKRN1, PPAR 22 activities with or without troglitazone were doubled compared with those of the control siRNA (Supplementary Figure 1b). To analyze the effects of MKRN1 on PPAR $\gamma$ in a more physiological context, we employed a $\mathrm{PC}-3$ human prostate cancer cell line, which endogenously expresses PPAR $\gamma .{ }^{28}$ On ablation of MKRN1, we observed increased levels of PPAR $\gamma$ protein levels, with no significant changes in mRNA levels (Figure 1a). Overall, these observations indicate that MKRN1 may negatively regulate PPAR $\gamma$ in post-translation.

MKRN1 functions as an E3 ligase of PPAR $\gamma$. The ability of MKRN1 to regulate PPAR $\gamma$ activities through post-translational regulation led us to examine the interactions between MKRN1 and PPAR $\gamma 2$. Two proteins that are exogenously expressed in 293T cells interact with each other (Figure 1b,
Supplementary Figure 2a). Interactions between endogenous PPAR $\gamma$ and MKRN1 were further observed using extracts of PC-3 or 3T3-L1 cells differentiated into adipocytes by DMI (a cocktail of dexamethasone, 3-isobutyl-1-methylxanthine, and insulin) treatment (Figures 1c and d). Finally, employing GST-pulldown assays using recombinant GSTPPAR 22 and MKRN1 translated in vitro, we were able to show that the two proteins interact directly (Figure 1e). Domain analyses using a truncated mutant of MKRN1 showed that the C-terminal region of MKRN1 was responsible for its interaction with PPAR 2 . On the other hand, $\mathrm{N}$-terminal region of PPAR $\gamma 2$ was required for its interaction with MKRN1 (Supplementary Figures 2b and c).

As MKRN1 is known as an E3 ligase, its ability to induce degradation of PPAR $\gamma 2$ was tested. ${ }^{21-25}$ With an increase of MKRN1 expressed in H1299 cells, the protein levels of PPAR $\gamma 2$ decreased (Figure 2a). Transient overexpression of MRKN1 in differentiated 3T3-L1 adipocytes suppressed the accumulation of endogenous PPAR $\gamma$, suggesting that MKRN1 induces PPAR $\gamma$ destabilization. However, the addition of proteasome inhibitor reverted the destabilization of PPAR $\gamma$. As expected, MKRN1 E3 ligase-defective mutant H307E did not have significant effects on PPAR $\gamma$ stability (Figure $2 b$ ). Treatment with MG132, a proteasome inhibitor, blocked MKRN1-mediated degradation of PPAR 2 , indicating that proteasome-dependent degradation is required in these processes (Figure 2c). When H307E, a MKRN1 E3 ligasedefective point mutant, was employed, it was able to bind to, but incapable of inducing degradation of, PPAR $\gamma 2$, indicating that E3 ligase activities of MKRN1 are involved in the degradation mechanism (Figure 2d, Supplementary Figure 3a). Using cycloheximide ( $\mathrm{CHX}$ ), an inhibitor of protein translation, MKRN1 was shown to reduce the half-life of PPAR 2 significantly, whereas H307E was not (Supplementary Figure $3 b$ ). These observations suggest that the ablation of MKRN1 increased the stability and thus the half-life of endogenous PPAR $\gamma$ protein in PC-3 cells (Supplementary Figure 3c).

To further confirm the activities of MKRN1 on PPAR 2 , ubiquitination analyses were carried out under denaturation conditions. His-Ub pulldown assays using $\mathrm{Ni}^{2+}-\mathrm{NTA}$ followed by immunoblotting of PPAR 2 showed that there was an increase of PPAR $\gamma 2$ ubiquitination in the presence of MKRN1 under treatment with MG132 (Figure 3a). On the other hand, the H307E mutant was not able to induce ubiquitination, suggesting that the E3 ligase activities of MKRN1 are required for PPAR $\gamma 2$ ubiquitination (Figure 3a). The ubiquitination analyses were extended by first immunoprecipitating PPAR $\gamma 2$, followed by immunoblotting to detect HA-Ub. These analyses also confirmed that only MKRN1 was able to induce PPAR 2 ubiquitination, but not H307E (Figure 3b). We detected residual amounts of ubiquitinated PPAR $\gamma$ in the absence of MKRN1. Based on these data, we cannot exclude the possibility that other E3 ligases are involved in the ubiquitination of PPAR $\gamma .{ }^{20}$ The levels of endogenous PPAR $\gamma$ ubiquitinated were further detected using a whole-cell lysate of differentiated 3T3-L1. The results showed that in the presence of stably expressed MKRN1, there was an increase of ubiquitinated endogenous PPAR $\gamma$ in differentiated 3T3-L1 cells, implicating MKRN1 as an E3 ligase of PPAR $\gamma$ (Figure 3c). Finally, using a cell-free 
a

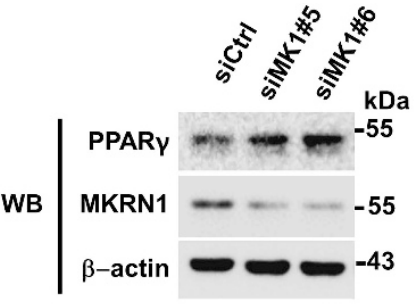

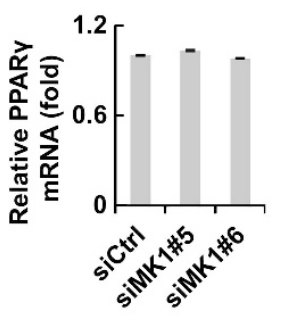

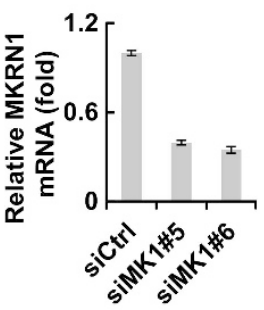

b

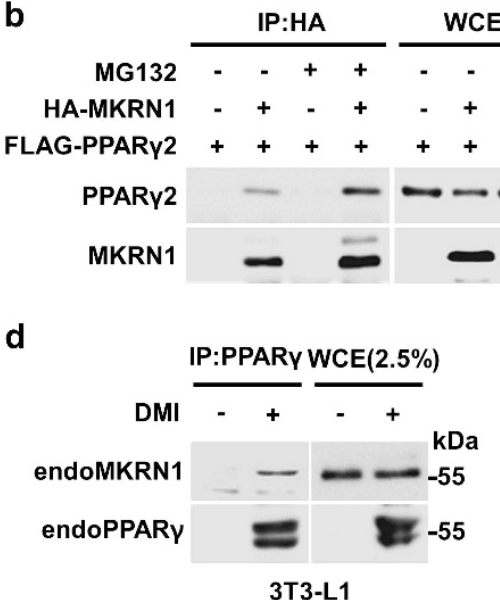

C e GST-PPARY2 - + ++ HA-MKRN1 + + + $+\mathrm{kDa}$

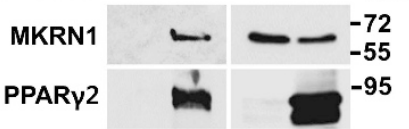

Figure 1 MKRN1 directly interacts with PPAR $\gamma$. (a) Effects of MKRN1 depletion on PPAR $\gamma$ protein and mRNA levels. PC-3 cells were transfected with the indicated siRNAs for $48 \mathrm{~h}$ and analyzed by western blotting or by qRT-PCR. (b) Interaction between exogenous MKRN1 and PPAR 2 . HEK293T cells were transfected with the plasmids expressing $3 \times$ FLAG-PPAR $\gamma 2$ (FLAG-PPAR $\gamma 2$ ) and HA-MKRN1 plasmids with or without $10 \mu \mathrm{M}$ MG132 for $6 \mathrm{~h}$. The transfected cells were then harvested and WCE were immunoprecipitated using $\alpha-\mathrm{HA}$ antibodies, followed by western blotting using $\alpha-\mathrm{FLAG}$ and $\alpha-\mathrm{HA}$ antibodies. Cell lysates ( $5 \%$ input) were used for western blotting. (c and $\mathbf{d}$ ) Interactions between endogenous (endo) MKRN1 and PPAR $\gamma$. PC-3 cell lysates were immunoprecipitated using $\alpha$-PPAR $\gamma$ or $\alpha$-IgG antibodies followed by western blotting using $\alpha$-PPAR $\gamma$ and $\alpha$-MKRN1 antibodies. Cell lysates (2.5\% input) were used for western blotting (c). WCE of 3T3-L1 undifferentiated or differentiated into adipocytes using DMl (dexamethasone +3 -isobutyl-1-methylxanthine + insulin) were immunoprecipitated using $\alpha$-PPAR $\gamma$ antibodies and detected as above. Cell lysates (2.5\% input) were used for western blotting (d). (e) Direct interaction between PPAR $\gamma 2$ and MKRN1 in vitro. In vitro translated MRKN1 was incubated with or without recombinant protein GST-PPAR $\gamma 2$, followed by GST pulldown using glutathione-Sepharose. The proteins were detected using $\alpha$-HA and $\alpha$-PPAR $\gamma$ antibodies. Input (5\%) were used for western blotting

a

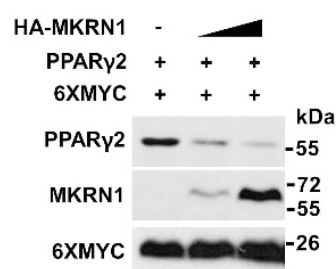

b

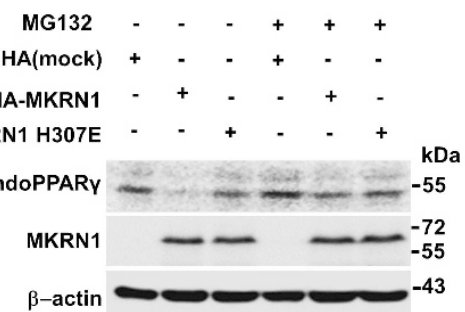

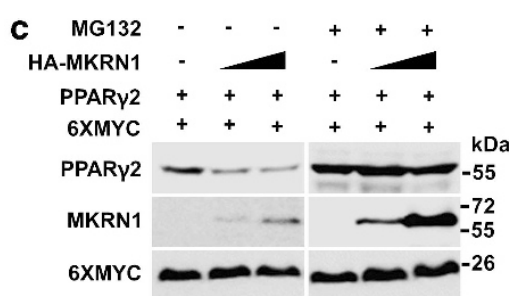

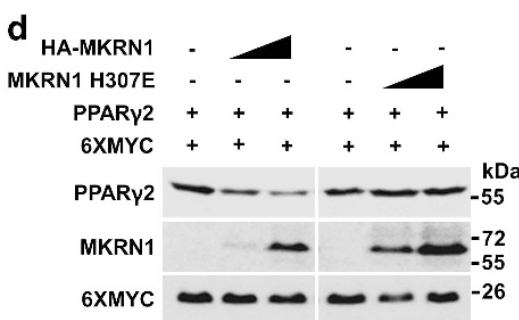

Figure 2 MKRN1 decreases PPAR 2 protein stability through its E3 ligase function. (a) PPAR 2 degradation by MKRN1. H1299 cells were transfected with the plasmids expressing PPAR $2(0.4 \mu \mathrm{g}), 6 \mathrm{XMYC}(0.3 \mu \mathrm{g})$ and increasing concentrations of HA-MKRN1 $(0.2$ and $0.4 \mu \mathrm{g})$, followed by western blotting. 6 XMYC was used as a transfection control. (b) Degradation of endogenous PPAR $\gamma$ by MKRN1. 3T3-L1 cells, differentiated with DMI for 2 days, were transfected with the plasmids expressing HA-MKRN1 and HA-MKRN1 H307E using a microporator in the presence or absence MG132. Cells were harvested $24 \mathrm{~h}$ after transfection and lysed, detected by western blotting using the indicated antibodies. (c) Effects of MG132 on MKRN1-mediated PPAR $\gamma 2$ degradation. $\mathrm{H} 1299$ cells, transfected with the plasmids as indicated above, were treated with $10 \mu \mathrm{M}$ MG132 for $6 \mathrm{~h}$. The proteins were detected by western blotting. (d) Effects of MKRN1 H307E mutant on PPAR 2 degradation. H1299 cells were transfected with the plasmids expressing PPAR $\gamma 2(0.4 \mu \mathrm{g})$, increasing amount of HA-MKRN1 or HA-MKRN1 H307E $(0.2$ and $0.4 \mu \mathrm{g})$. WCE were detected by western blotting, as described above 

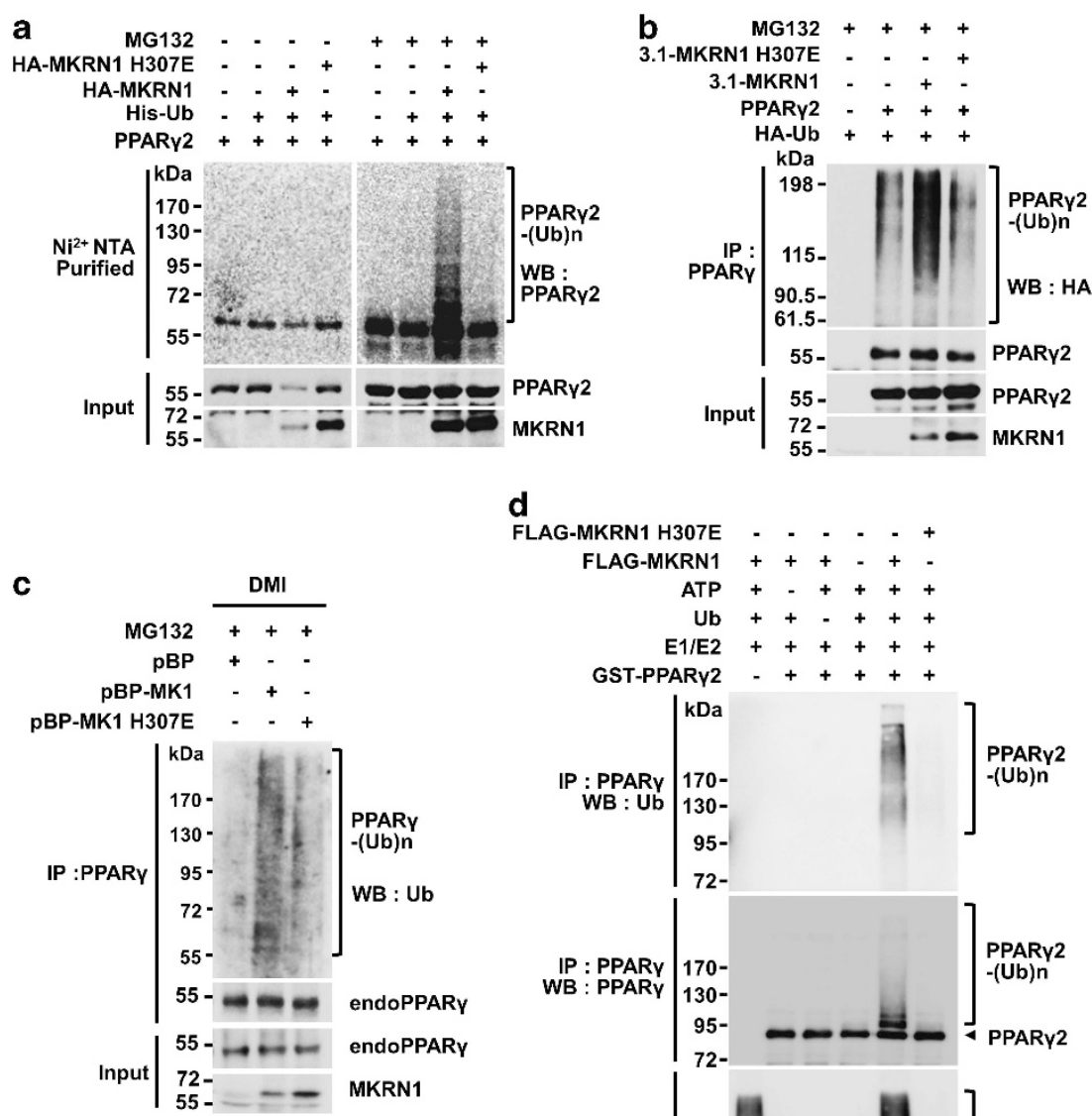

d

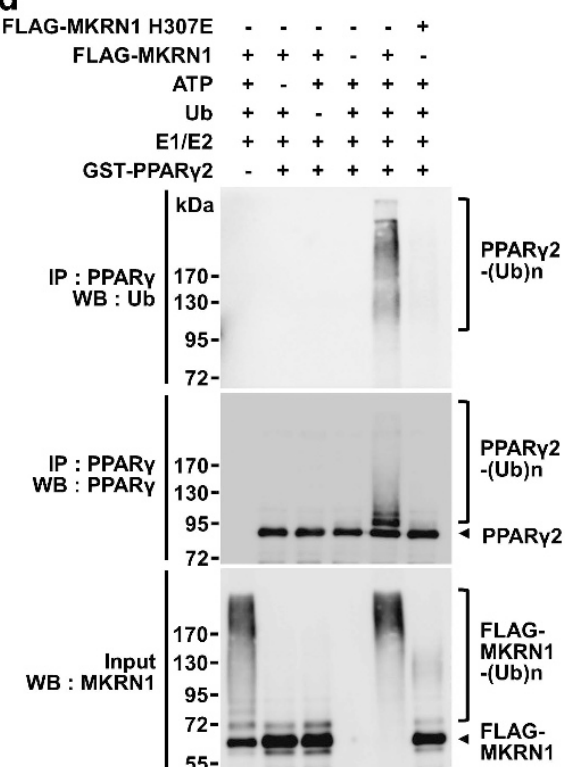

Figure 3 MKRN1 mediates ubiquitination of PPAR $\gamma 2$ through E3 ligase activity. (a) Ubiquitination of PPAR $\gamma 2$ by MKRN1 and MKRN1 H307E mutants. H1299 cells were transfected with the indicated plasmid in the presence or absence of MG132. His-Ub conjugated proteins were pulled-down using Ni ${ }^{2+}{ }_{-N T A}$ beads and detected using $\alpha$ PPAR $\gamma$ or $\alpha$-HA antibodies. (b) PPAR $\gamma 2$ ubiquitination by active MKRN1 E3 ligase. H1299 cells, transfected as indicated, were immunoprecipitated with monoclonal $\alpha$-PPAR $\gamma$ antibodies, followed by western blotting as described above. (c) Ubiquitination of endogenous PPAR $\gamma$ by MKRN1. 3T3-L1 stable cell lines expressing pBabe-Puro-MKRN1 (pBP-MK1) and pBabe-Puro-MKRN1 H307E (pBP-MK1 H307E) were differentiated by treatment with DMl. Cells were treated with MG132 for $6 \mathrm{~h}$ before harvest and lysed, followed by immunoprecipitation using $\alpha$-ubiquitin antibodies and western blotting. (d) Ubiquitination of PPAR 2 by MKRN1 in vitro. Purified recombinant GST-PPAR 2 was incubated with E1, E2, ubiquitin (Ub) and ATP in the absence and presence of FLAG-MKRN1 wild-type or H307E mutant as indicated. Reactions were boiled in 1\% SDS to disrupt protein interaction, followed by immunoprecipitation using $\alpha$-PPAR $\gamma$ antibodies. Ubiquitination of PPAR $\gamma$ was analyzed by western blotting using $\alpha$-Ub or $\alpha$-PPAR $\gamma$ antibodies. For input, ubiquitin reactions were directly subjected to western blotting using $\alpha$-MKRN1 antibodies

ubiquitination assay system, MKRN1 was shown to induce ubiquitination of recombinant PPAR 2 , whereas MKRN1 H307E was not (Figure 3d). GST was used as a control (Supplementary Figure 7a). We observed a similar effect of MKRN1 on PPAR $\gamma 1$ ubiqutination and degradation, suggesting that both PPAR $\gamma 1$ and PPAR $\gamma 2$ are substrates of MKRN1 (Supplementary Figures $4 a-c$ ).

Previous reports indicated that the presence of PPAR $\gamma 2$ agonist expedites the degradation process of PPAR $\gamma 2 .{ }^{17}$ To verify whether PPAR $\gamma 2$ agonist facilitates MKRN1-dependent degradation processes, troglitazone was included in the assays. On treatment with troglitazone, there was an increase in MKRN1 and PPAR $\gamma 2$ interaction, with more efficient MKRN1-dependent degradation of PPAR $\gamma 2$ (Supplementary Figures $5 \mathrm{a}$ and $\mathrm{b}$ ). In agreement with these data, PPAR $\gamma 2$ ubiquitination was further increased by the agonist (Supplementary Figure 5c). Finally, using wild type or MKRN1 null MEFs, we showed that the presence of PPAR 2 agonists such as rosiglitazone, troglitazone, or GW7845 stimulated PPAR 2 destabilization in wild-type MEFs, whereas these effects were not clearly observed in MKRN1 knockout MEFs (Supplementary Figure $5 \mathrm{~d}$ ). These results suggest that agonist-stimulated destabilization of PPAR 2 requires the presence of MKRN1. These agonists did not affect MKRN1 levels, as shown in Supplementary Figure $5 \mathrm{~d}$. In summary, on interacting with PPAR $\gamma$, MKRN1 was able to induce the ubiquitination and degradation of PPAR $\gamma$, and these MKRN1mediated processes were facilitated by the presence of a PPAR $\gamma$ agonist.

Two lysines of PPAR $\gamma$ located at the putative nucleus localization signal (NLS) are targeted for ubiquitination by MKRN1. PPAR $\gamma$ is a nuclear receptor with a putative NLS region located between amino-acid regions 181 and $224 .{ }^{29,30}$ 
Our immunocytochemistry analyses showed that $\sim 80 \%$ of $\mathrm{H} 1299$ cells exhibited the nucleus-only localization of PPAR $\gamma 2$, as previously reported (Supplementary Figure 6). ${ }^{31}$ Positively charged lysine sites in the NLS are commonly considered to have important roles in the nuclear localization of various proteins. ${ }^{29-32}$ When two lysines that are consecutively located at 184 and 185 sites in the NLS region were mutated to alanines (2KA), $\sim 30 \%$ of the cells showed nuclear localization of PPAR $\gamma 2$, whereas the rest displayed nuclear/ cytosol localization (Supplementary Figure 6). However, when the two lysines were mutated to arginines (2KR), no significant changes of PPAR $\gamma 2$ localization were observed (Supplementary Figure 6). On co-expression of MKRN1 and PPAR $\gamma 2$ or $2 \mathrm{KR}$, we observed the nuclear/cytoplasmic localization of PPAR $\gamma 2$ in $70 \%$ of cells expressing both proteins (Supplementary Figure 6). These observations suggest that MKRN1 might mask the NLS function of PPAR $\gamma 2$ in a way that blocks its localization into the nucleus. As expected, 2KA was able to bind to MKRN1 as the wild type (Figure $4 \mathrm{a}$ ). One interesting observation was that this protein was protected from MKRN1-mediated ubiquitination and degradation, indicating that these two lysines might be targeted for ubiquitination by MKRN1 (Figures 4b-d). Overall, MKRN1-mediated ubiquitination of PPAR $\gamma 2$ at lysines 184 and 185 located at NLS may be necessary for its ubiquitination and subsequent degradation (Figure 4e). A cell-free ubiquitination system was employed to further confirm whether lysines 184 and 185 are actually targeted for ubiquitination by MKRN1. The 2KA mutant was not ubiquitinated by MKRN1, suggesting that these two sites are indeed targeted for ubiquitination (Supplementary Figure 7b). As MKRN1 was able to induce translocation of both wild-type PPAR $\gamma 2$ and $2 K R$ mutants into the cytoplasm, interaction between the two proteins seems to be required for translocation of PPAR $\gamma$ by MKRN1, rather than the ubiquitination process.

MKRN1 suppresses adipocyte differentiation. As the forced expression of PPAR $\gamma$ in fibroblastic cells can induce adipogenesis, we investigated the physiological effects of MKRN1 in adipocyte differentiation. The role of MKRN1 as a negative regulator of PPAR $\gamma$ was analyzed by adipocyte differentiation from 3T3-L1 pre-adipocytes stably overexpressing MKRN1 or H307E. Overexpression of MKRN1 using a retrovirus suppressed the adipogenesis of 3T3-L1 induced by DMI treatment, as assessed using Oil-Red-O staining and morphological analysis (Figure $5 \mathrm{a}$ and Supplementary Figure 8a). Overexpressed MKRN1 consistently suppressed the expressions of adipogenic markers, such as aP2, C/EBP $\alpha$, and CD36, as well as PPAR $\gamma$ (Figures 5b and c). Overexpressed H307E did not exhibit any noticeable effects on adipogenes, which corroborates the data shown above (Figures 5a-c and Supplementary Figure 8a). To further confirm whether overexpressed MKRN1 could a

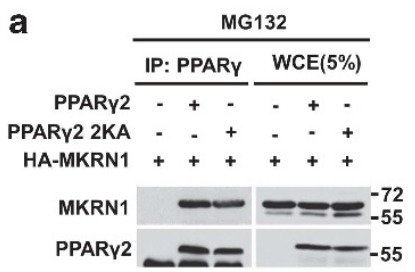

b

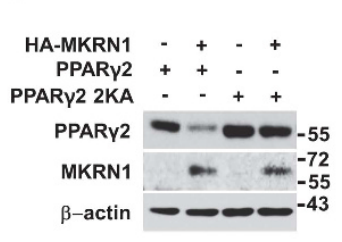

d

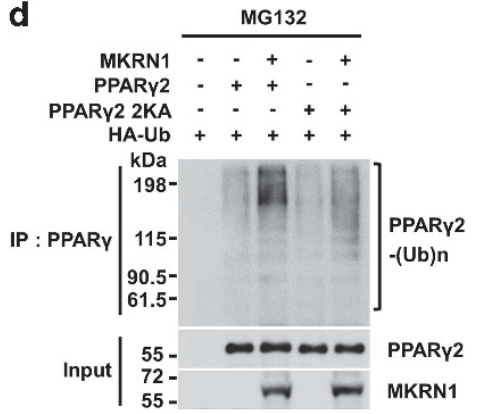

C

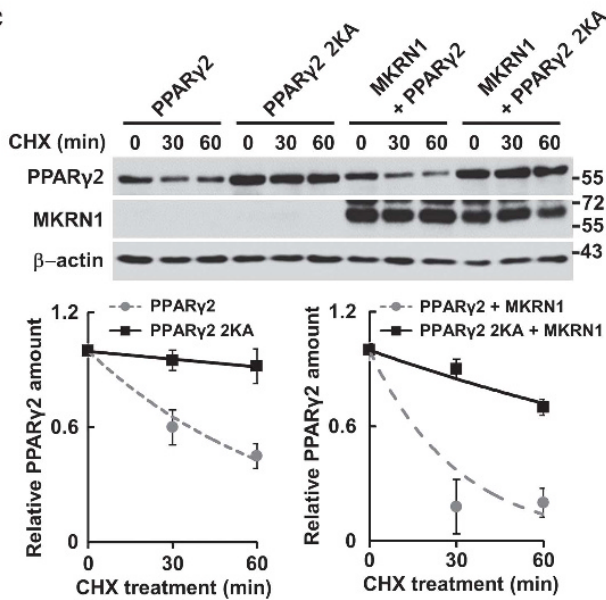

e

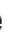

PPARY2

MKRN1 binding region

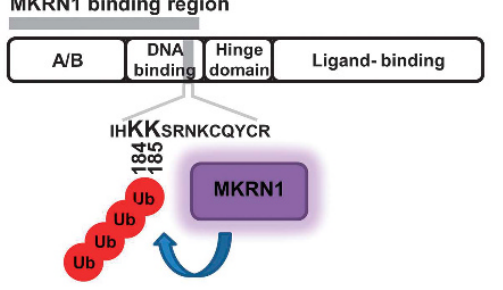

Figure 4 Two lysine residues of PPAR 2 (184 and 185) are targeted for ubiquitination by MKRN1. (a) Interaction between PPAR 2 point mutant (2KA) and MKRN1. HEK293 T cells were transfected with the plasmids expressing PPAR $\gamma 2$ WT and PPAR 22 2KA mutant alone or with HA-MKRN1. WCE were immunoprecipitated with $\alpha$-HA antibodies followed by western blotting using $\alpha$-HA or $\alpha$-PPAR $\gamma$ antibodies. Cell lysates ( $5 \%$ input) were used for western blotting. (b) Effects of MKRN1 on protein stability of PPAR $\gamma 2$ 2KA mutant. H1299 cells transfected with plasmids expressing the indicated proteins were detected by western blotting, as indicated above (Figure 2a). (c) Half-life of PPAR 22 2KA mutant in the presence of MKRN1. H1299 cells were transfected with plasmids expressing indicated proteins followed by treatment with $50 \mu \mathrm{g} / \mathrm{ml} \mathrm{CHX}$ for up to 60 min. WCE were perceived by western blotting and their levels measured as in Supplementary Figure 3b. (d) PPAR 22 2KA ubiquitination by MKRN1. H1299 cells were transfected with the plasmids expressing MKRN1, PPAR $\gamma 2$, PPAR $\gamma 2$ 2KA, or HA-Ub. WCE were immunoprecipitated with monoclonal $\alpha$-PPAR $\gamma$ antibodies, followed by western blotting, as described in Figure $3 \mathrm{~b}$. (e) Schematic of PPAR $\gamma 2$ ubiquitination target sites 


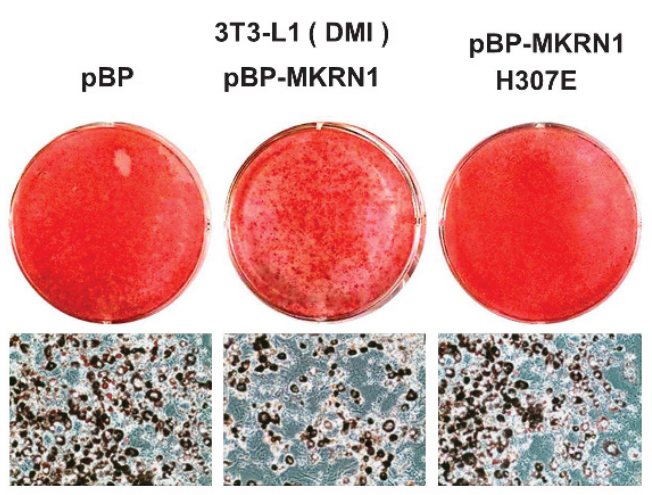

d

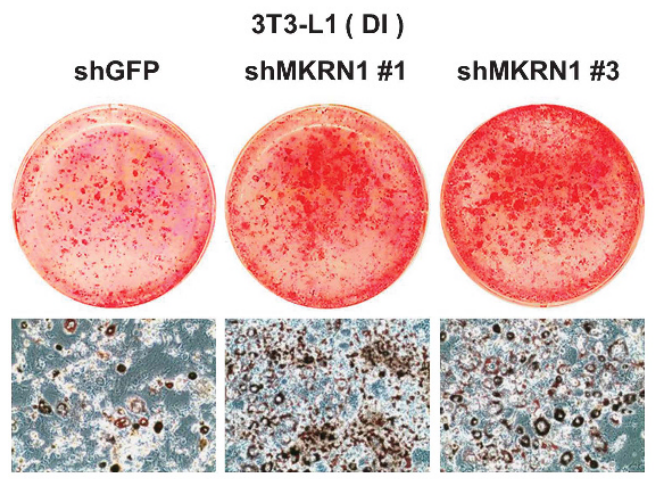

b

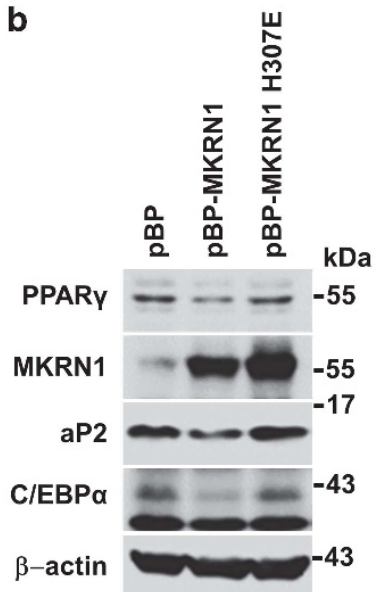

e

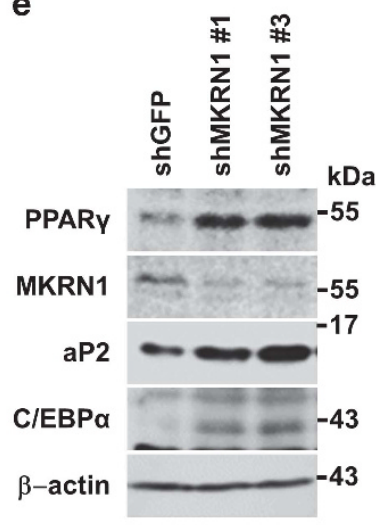

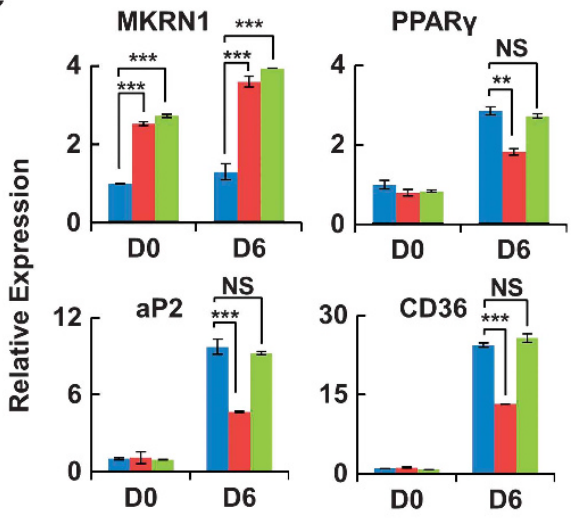

" pBP $\approx$ pBP-MKRN1
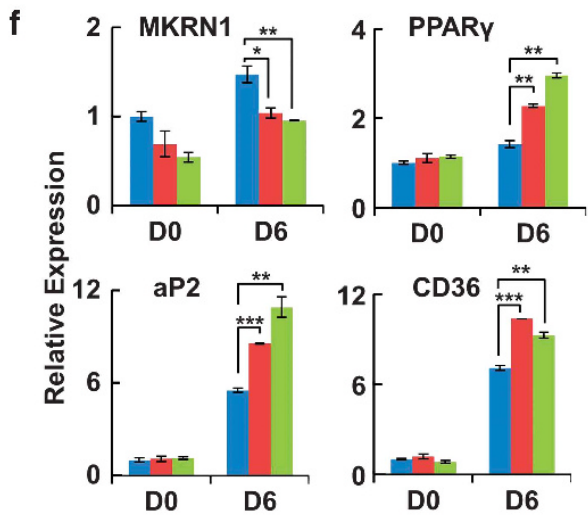

shGFP shMKRN1\#1 shMKRN1\#3

Figure 5 MKRN1 reduces PPAR $\gamma$ protein levels and inhibits adipocyte differentiation in 3T3-L1 cells. (a) Effects of MKRN1 overexpression on 3T3-L1 adipocyte differentiation. Stable 3T3-L1 cell lines overexpressing MKRN1 (pBP-MKRN1) or its H307E mutant (pBP-MKRN1 H307E) using retroviral vectors were treated with DMI for 6 days and their adipocyte differentiation was detected using Oil-Red-O staining. 3T3-L1 cells retrovirally transduced with pBabe-puro empty vector (pBP) were used as control cells. (b) Effects of MKRN1 overexpression on the levels of PPAR $\gamma$ and its target proteins. The WCE described above were analyzed using antibodies against the proteins indicated. (c) Levels of adipocyte differentiation markers in 3T3-L1 cells expressing MKRN1. mRNA levels of cells indicated above were measured by qRT-PCR analysis using primers targeted for MKRN1, PPAR $\gamma$, aP2 and CD36 mRNAs. Data are presented as mean \pm S.D.; $n=3$ with ${ }^{* \star} P<0.01$ and ${ }^{* * *} P<0.001$ compared with each lane. (d) Effects of MKRN1 depletion on adipogenesis of 3T3-L1. Stable cell lines constitutively depleted of MKNR1 were constructed using a lentivirus expressing shRNA for GFP or mouse MKRN1 (shGFP, shMKRN1 \#1, shMKRN1 \#3, respectively). The adipogeneses of these three cell lines were induced using DI (dexamethasone + insulin) for 6 days. Cells were stained and detected as described above. (e) MKRN1 knockdown increases the levels of adipogenic proteins. WCE from above were analyzed as above. (f) Effects of MKRN1 depletion on mRNA levels of the adipogenic markers. The mRNA of adipogenic markers were analyzed as described above. Data are presented mean \pm S.D.; $n=3$ with ${ }^{*} P<0.05,{ }^{* *} P<0.01$ and ${ }^{* * *} P<0.001$ compared with each lane

suppress adipocyte differentiation by specifically targeting PPAR $\gamma$ for destabilization, we performed rescue experiments by expressing PPAR $\gamma$ in the presence of MKRN1. We observed that overexpression of PPAR $\gamma$ was able to overcome MKRN1-dependent suppression of adipocyte differentiation, suggesting that MKRN1 could target PPAR $\gamma$ as its substrate (Figure 6). The effects of MKRN1 on adipocyte differentiation were further confirmed under MKRN1depleted conditions. To maximize the effects of MKRN1 depletion using shMKRN1 \#1 and \#3 on adipocyte differentiation, we applied DI instead of DMI, which is known to induce less-efficient adipocyte differentiation. ${ }^{33,34}$ In both stable cell lines, we were able to observe effective differentiation of pre-adipocytes with greater expressions of PPAR $\gamma, a P 2$, and its other targets (Figures $5 \mathrm{~d}$ and e and Supplementary Figure 8b). Consistently, the real-time reverse-transcription PCR ( $\mathrm{QRT}-\mathrm{PCR}$ ) data indicated that the adipogenic markers, including PPAR $\gamma$, aP2, and CD36, were all increased under MKRN1 depletion (Figure 5f). The effects of MKRN1 on adipocyte differentiation were also observed in mesenchymal C3H10T1/2 cells. MKRN1 or pBabe empty vector was stably overexpressed and stimulated into adipocytes with the addition of DMI and PPAR $\gamma$ agonists, GW7845. Consistent with the effects in 3T3-L1 cells, C3H10T1/2 cells stably expressing MKRN1 decreased PPAR $\gamma$ protein expression, resulting in less lipid accumulation compared with control cells (Supplementary Figures 9a and b). Gene expression analyses also showed decreased expression of PPAR $\gamma$ and its target aP2 mRNA in cells stably expressing MKRN1 (Supplementary Figure 9c). To address the roles of endogenous MKRN1 in C3H10T1/2 cells, stable cells expressing shMKRN1 \#3 or GFP were generated. Compared with control cells, stable C3H10T1/2 cells expressing shMKRN1 \#3 exhibited markedly increased lipid accumulation and expression of adipocyte markers (Supplementary Figures 10a-c). 

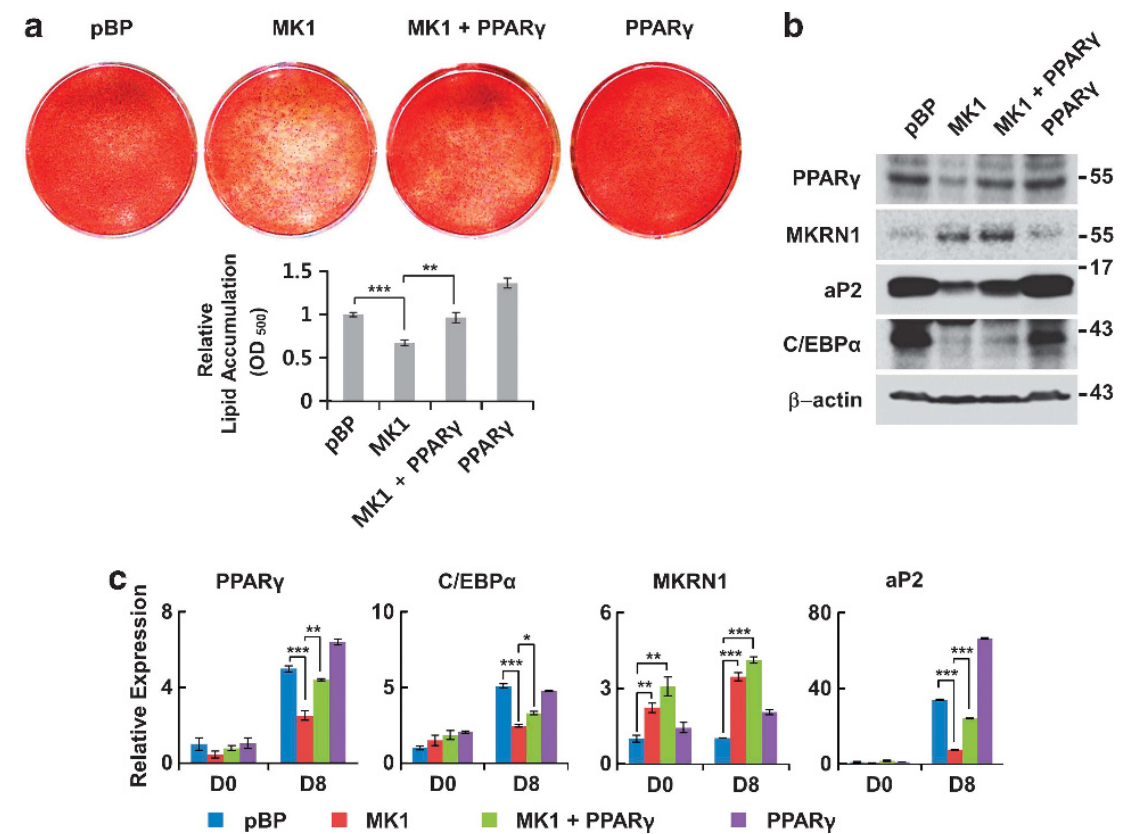

Figure 6 MKRN1-dependent suppression of adipogenesis was rescued by the expression of PPAR $\gamma$ in 3T3-L1 cells. (a) Effects of overexpressed PPAR $\gamma$ on MKRN1dependent suppression of adipocyte differentiation. Stable cell lines retrovirally overexpressing MKRN1 (MK1), PPAR $\gamma$ or MKRN1 + PPAR $\gamma$ (MK1 + PPAR $\gamma$ ) were treated with DMI for 6 days, and adipocyte differentiation was detected using Oil-Red-O staining. 3T3-L1 cells transfected with retrovirus containing pBabe-puro empty vector (pBP) were used as control cells. Oil-Red-O staining extractions were performed as indicated above. (b and $\mathbf{c}$ ) Expression patterns of PPAR $\gamma$ and its targets. WCE from above were detected using antibodies against proteins indicated (b). mRNA levels of adipogenic markers in 3T3-L1 stable cell lines were analyzed by qRT-PCR method using indicated primers (c). Data are presented as mean \pm S.D.; $n=3$ with ${ }^{\star} P<0.05,{ }^{* \star} P<0.01$ and ${ }^{* \star} P<0.001$ compared with each lane

To further confirm the effects of MKRN1 in adipogenesis shown above, potentials of MKRN1 knockout or wild-type MEFs for adipocyte differentiation were compared. Similar to the increased differentiation in MKRN1-silenced 3T3-L1 and C3H10T1/2 cells, primary MKRN1 - / - MEFs showed robust increases in lipid accumulation and morphological differentiation when compared with WT MEFs (Figures $7 \mathrm{a}-\mathrm{C}$ ). There were also significant increases in protein and mRNA levels of PPAR $\gamma, a P 2, \mathrm{C} / \mathrm{EBP} \alpha$, and CD36 in the MKRN1 - / - cells (Figures $7 d$ and $e$ ). As expected, the expressions of C/EBP $\beta$ and $\mathrm{C} / \mathrm{EBP} \delta$ were not affected in the MKRN1 $1-/$ - cells, suggesting that there are PPAR $\gamma$-specific effects of MKRN1 in adipogenesis (Figures $7 d$ and e). Confirming these observations, the ubiquitination status of endogenous PPAR $\gamma$ was weaker under MKRN1 - / - MEFs, suggesting that MKRN1 is necessary for PPAR $\gamma$ ubiquitination (Figure 7f). Overall, our data demonstrate that MKRN1 can negatively control adipocyte differentiation by suppressing PPAR $\gamma$ function posttranslationally.

\section{Discussion}

The combined trends of increased caloric intake and decreased physical exercise have contributed to the increasing prevalence of obesity and related insulin resistance in industrialized populations. Insulin resistance is associated with increased risks of type 2 diabetes and cardiovascular disease. At the crossroads of obesity, insulin resistance, and cardiovascular disease lies the nuclear receptor PPAR $\gamma .{ }^{35-38}$ Therefore, better understanding of PPAR biology is of scientific and therapeutic importance.
As PPAR $\gamma$ functions as a master regulator of adipogenesis and is a critical factor associated with various metabolic diseases, amounts of PPAR $\gamma$ should be tightly controlled at the transcriptional and post-translational levels. ${ }^{5,8,35}$ Transcriptional pathways that control the expression of PPAR $\gamma$ have been extensively studied;:76 but the stability of short-lived PPAR $\gamma$, which is probably controlled by post-translational modification, remains incompletely understood. Previous studies showed that PPAR $\gamma$ is polyubiquitinated and degraded in a proteasome-dependent manner. ${ }^{17}$ Ubiquitin ligase Siah2 was recently identified for its inhibitory actions on PPAR $\gamma$ activity, further suggesting that ubiquitin ligase is an important regulator of PPAR $\gamma$ activity and adipogenesis. However, Siah2 does not appear to directly interact with PPAR $\gamma$, but instead a nuclear receptor corepressor, NCoR, has been suggested as a target of Siah2, indicating that another E3 ligase(s) exists for ubiquitination of PPAR ${ }^{19}$

In this study, we propose MKRN1 as a direct E3 ligasetargeting PPAR $\gamma$. MKRN1 reduces PPAR $\gamma$ protein levels through direct interaction. Two lysine residues at 184 and 185 in the DNA-binding domain of PPAR $\gamma$ are critical sites for MKRN1-mediated ubiquitination. Interestingly, these two lysine residues are conserved in mammals, birds, reptiles, amphibians, and fishes, implying an evolutionarily conserved role for MKRN1 in PPAR $\gamma$ regulation. Overexpression and knockdown studies indicate that MKRN1 is a negative regulator of PPAR $\gamma$, decreasing PPAR $\gamma$ protein levels. MEFs isolated from MKRN1 - / - embryos further demonstrate the regulatory roles of MKRN1 in PPAR $\gamma$-mediated adipogenesis. Thus, our results demonstrate that MKRN1 is a novel 
a

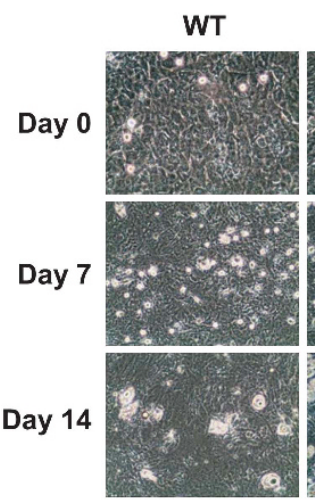

d

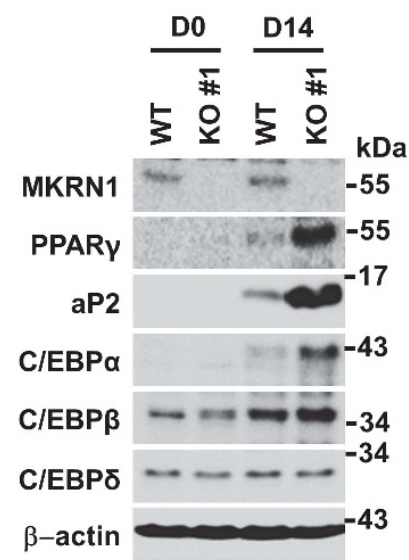

b

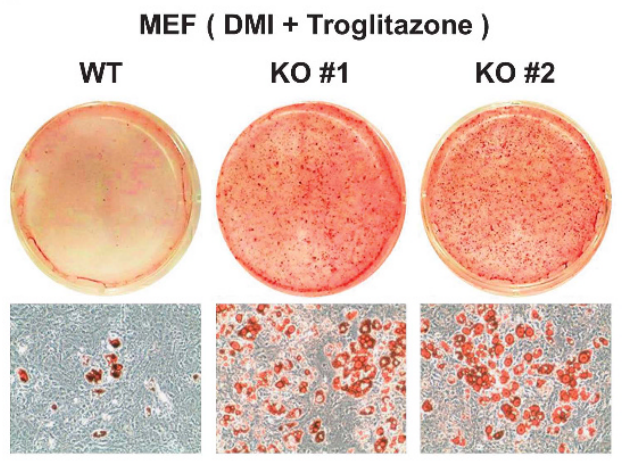

Oil-Red-O staining

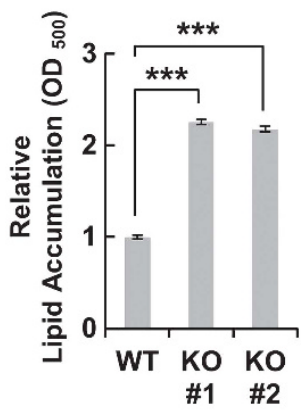

e

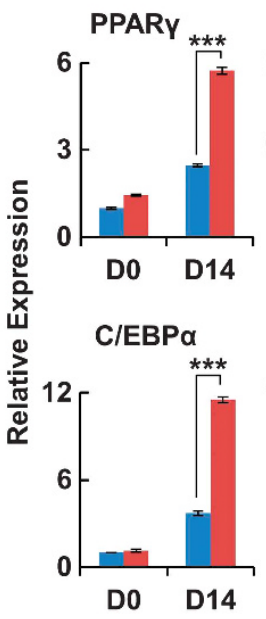

aP2

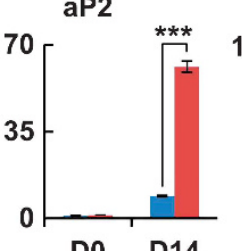

C/EBP $\beta$

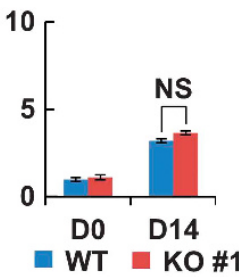

f

CD36
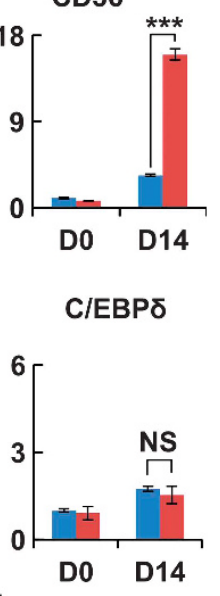

$\mathrm{DMI}+\mathrm{TrO}+\mathrm{MG}$

Figure 7 MKRN1 knockout MEFs exhibit the increased potential for adipocyte differentiation. (a and b) Adipogenesis of MKRN1 knockout MEFs. MKRN1 knockout (KO \#1 and $\mathrm{KO}$ \#2) and wild-type MEFs, cultured in medium containing DMI and troglitazone from 0 to 14 days, were photographed as described above (a). Differentiated MEF cells were detected using Oil-Red-O staining (b). (c) The Oil-Red-O dye was extracted using isopropanol and measured at $500 \mathrm{~nm}$. Data represent mean \pm S.D.; $n=3$ with ${ }^{* * *} P<0.001$. See Materials and methods for details. (d) Protein levels of PPAR $\gamma$ and its target proteins in wild type and MKRN1 knockout MEFs. MKRN1 knockout MEFs displayed increased levels of PPAR $\gamma$ with its target proteins. WCE of MKRN1 knockout and wild-type MEFs were analyzed as described in Figure 5b. (e) mRNA levels of PPAR $\gamma$ and its target proteins in wild type and MKRN1 knockout MEFs. MKRN1 knockout MEFs increase in mRNA levels of the adipogenic markers. The mRNA of adipogenic markers and upstream of PPAR $\gamma$ were analyzed by qRT-PCR analysis as described in Figure 5c. Data are mean \pm S.D.; $n=3$ with ${ }^{* \star *} P<0.001$ compared with each lane. (f) Reduction of endogenous PPAR $\gamma$ ubiquitinationin MKRN1 knockout MEFs. MKRN1 WT and knockout MEFs were differentiated to adipocytes using DMI and troglitazone (DMI + tro) for 14 days. Subsequently, WT and knockout MEFs were treated with $10 \mu \mathrm{M}$ MG132 (MG) for $6 \mathrm{~h}$ before harvest and lysed, followed by immunoprecipitation using $\alpha$-PPAR $\gamma$ antibodies and western blotting under denaturating conditions

regulator of $\operatorname{PPAR} \gamma$, catalyzing polyubiquitination and subsequent degradation of PPAR $\gamma$.

Previous studies have implicated that an elusive E3 ligase responsible for PPAR $\gamma$ degradation is tightly linked to PPAR $\gamma$ activation on ligand binding. ${ }^{17}$ Therefore, we investigated whether the regulatory actions of MKRN1 could be affected by PPAR $\gamma$ activation. The regulatory activities of MKRN1 on PPAR $\gamma$ appear to be influenced by ligand binding, as treatment with troglitazone enhanced the association between the two proteins and increased the ubiquitination of PPAR $\gamma$, confirming previous findings (Supplementary Figures 5a-d). ${ }^{17,19}$ How does PPAR $\gamma$ activation by agonists facilitate MKRN1 and PPAR $\gamma$ interaction and subsequently increase the degradation of PPAR $\gamma$ ? One may speculate that the activation of PPAR $\gamma$ causes a conformational shift toward the higher incidence of MKRN1 binding with PPAR $\gamma$, followed by cytoplasmic relocation and degradation. How the regulatory functions of MKRN1 act on PPAR $\gamma$ in a ligand-dependent manner remains an intriguing question for future studies.

In this study, we identified the functions of MKRN1 in posttranslational modification of PPAR $\gamma$ and adipocyte differentiation. Interestingly, however, MKRN1 is expressed in multiple tissues, suggesting functions in other cell types. Whether MKRN1 leads to the suppression of PPAR $\gamma$ in non-adipose tissues and similar modulations of other nuclear receptor families associated with metabolic disease are intriguing questions. Further studies are necessary to evaluate the effects of MKRN1 in non-adipose tissues and other targets for degradation.

Our results also suggest that MKRN1 could be a novel therapeutic target for obesity and diabetes. Further studies of MKRN1 activities and cellular and pathological effects in vivo are of great importance for finding novel therapeutic 
approaches to obesity-related diseases. The generation and characterization of tissue-specific knockout mice will be required to determine the roles of MKRN1 in obesity and related metabolic diseases. It will be interesting to explore whether MKRN1 and its mechanisms can be exploited to develop new therapeutic interventions for the treatment of obesity and metabolic diseases in the future.

\section{Materials and Methods}

Plasmids. pcDNA3.1-PPAR $\gamma 2$ was generated from pBabe-puro-PPAR $\gamma 2$, which was described previously. ${ }^{34}$ pcDNA3.1-PPAR 2 2KA and pcDNA3. 1-PPAR $\gamma 2$ 2KR mutants were produced using site-directed mutagenesis methods, as provided by the manufacturer (Takara Bio, Shiga, Japan). The primers used for these constructs are as follows: 2KA-5'-AACTGCCGGATCCACGCAGCAGCAAG AAATAAATGTCAG-3' (forward), 2KA-5'-CTGACATTTATTTCTTGCTGCTGCGTG GATCCGGCAGTT-3' (reverse), 2KR-5'-AACTGCCGGATCCACAGGAGGAGTAG AAATAAATGTCAG-3' (forward), 2KR-5'-CTGACATTTATTTCTACTCCTCCTGTG GATCCGGCAGTT-3' (reverse). MKRN1 constructs (pcDNA3-HA-MKRN1 WT/H307E, pcDNA3.1-MKRN1 WT/H307E, pcDNA3-FLAG-MKRN1 WT/H307E) were described previously. ${ }^{22}$ pcDNA3-HA-MKRN1 WT and H307E mutant were subcloned into pBabe-Puro. pcDNA3-His-Ub and pHM6-HA-Ub was previously described. ${ }^{39,40}$ pTK-PPREx3-luc was kindly provided by HW Lee (Yonsei University, Seoul, Korea).

Antibodies and chemicals. Western blotting and immunoprecipitation data analysis were performed using the following antibodies: PPAR (mouse sc-7273X, rabbit sc-7196X, Santa Cruz Biotechnology, Santa Cruz, CA, USA), C/EBP $\alpha$ (rabbit sc-61X, Santa Cruz Biotechnology), C/EBP $\beta$ (rabbit sc-150X, Santa Cruz Biotechnology), C/EBP $\delta$ (rabbit sc-636X, Santa Cruz Biotechnology), aP2 (goat sc-18661, Santa Cruz Biotechnology), HA (mouse sc-7392, rabbit sc-805, Santa Cruz Biotechnology), FLAG (mouse F3165, rabbit F7425; Sigma-Aldrich, St. Louis, MO, USA), GFP (sc-8334, Santa Cruz Biotechnology), $\beta$-actin (A5316; Sigma-Aldrich), normal mouse lgG (sc-2025; Santa Cruz Biotechnology), normal goat IgG (sc-2028; Santa Cruz Biotechnology), myc (sc-40, Santa Cruz Biotechnology), MKRN1 (A300-990A; Bethyl Laboratories, Montgomery, AL, USA), and HRPconjugated- $\alpha$-UbFK2 (PW0150, Enzo Life Sciences, Farmingdale, NY, USA). CHX (C4859), N-ethylmaleimide (NEM, E3876) and dimethyl sulfoxide (DMSO, D8418), dexamethasone (D1756), IBMX (15879), Troglitazone (T2573), Rosiglitzaone (R2408), and Oil-Red-O (O0625) were purchased from Sigma-Aldrich. Insulin (11 376497 001) was purchased from Roche (Mannheim, Germany). GW7845 was kindly provided by T Willson (GlaxoSmithKline, QC, Canada).

Transfection. H1299 and PC-3 cells were transfected using Lipofectamine 2000 according to the manufacturer's instructions (Invitrogen, Carlsbad, CA, USA). Polyethyleneimine (PEI, Sigma-Aldrich) was used for the transfection of HEK293T cells. 3T3-L1 cells were transfected using a microporator (Neon Transfection System, Invitrogen). Lipofectamin RNAiMax (Invitrogen) was used for siRNA transfection. MKRN1 siRNAs were previously described (the siRNA sequences are as follows: \#5- 5'GGCGAAGCTGAGTCAAGAA-3' and \#6- 5'-GGATCCTCTCC AACTGCAA- $\left.3^{\prime}\right){ }^{22}$

Cell culture and adipocyte differentiation. H1299 (human lung carcinoma cell line) and HEK293T (human kidney cancer cell line) were cultured in DMEM containing 10\% FBS (Gibco, Invitrogen). Adipocytes were differentiated from 3T3-L1 pre-adipocyte cells, mesenchymal C3H10T1/2 cells, or mouse MEFs. Briefly, 3T3-L1 cells were maintained in DMEM with $10 \%$ bovine serum for 3-5 days, and confluent cells differentiated in DMEM containing $10 \% \mathrm{FBS}$, dexamethasone (1 $\mu \mathrm{M})$, 3-isobutyl-1-methylxanthine (IBMX; $520 \mu \mathrm{M})$, and insulin $(1 \mu \mathrm{g} / \mathrm{ml})$ (DMl mixture) for 2 days. The cells were then cultured in DMEM containing $10 \%$ FBS and insulin $(5 \mu \mathrm{g} / \mathrm{ml})$ for 2 additional days. MEFs and C3H10T1/2 cells were cultured in DMEM with $10 \%$ FBS for $3-5$ days, and confluent cells differentiated in DMEM containing 10\% FBS, dexamethasone $(1 \mu \mathrm{M})$, 3-isobutyl-1-methylxanthine (IBMX; $520 \mu \mathrm{M})$, insulin $(10 \mu \mathrm{g} / \mathrm{ml}$; (DMI mixture) and TZD ( $1 \mu \mathrm{M}$, Troglitazone and GW9544) for 2-3 days. The cells were further cultured in DMEM containing $10 \% \mathrm{FBS}$, insulin $(10 \mu \mathrm{g} / \mathrm{ml})$, and TZD $(1 \mu \mathrm{M})$ for an additional 6-8 days. Differentiated cells were detected using Oil-Red-O staining, as previously described (O0625, Sigma-Aldrich). ${ }^{41}$ The stained cells were photographed using CKX41SF (Olympus, Tokyo, Japan). The dye from Oil-Red-O staining were extracted using isopropanol, followed by optical density measurement at $500 \mathrm{~nm}$ using Benchmark Plus (Bio-Rad Inc., US).

Generation of primary MEFs. MKRN1 knockout MEFs were generated as previously described using MKRN1 C57BL/6J mice. ${ }^{25}$ Briefly, at E13.5 day, embryos were removed from pregnant female mice and ground using blades. Ground embryos were incubated in $3 \mathrm{ml}$ of trypsin-EDTA $37^{\circ} \mathrm{C}$ for $20 \mathrm{~min}$, transferred to $150-\mathrm{mm}$ culture dishes, and incubated at $37^{\circ} \mathrm{C}$ in $20 \mathrm{ml}$ of complete media. After $8 \mathrm{~h}$, the medium was changed to fresh DMEM containing $10 \% \mathrm{FBS}$. Once the cells were $70-80 \%$ confluent, they were maintained at $1: 3$ ratios.

Generation of MKRN1 overexpression and knockdown stable cell lines. All short-hairpin RNA (shRNA) constructs were purchased from Sigma-Aldrich. The sequences are as follows: mouse MKRN1\#1-5'-GCG AGATGTTGCTTATGCTTT-3', MKRN1\#2-5'-CCAGAGGTCACAGCACATAAA-3', MKRN1\#3-5'-GAGTGGGACTTGTTTCACGAT-3'. 3T3-L1 cells stably expressing control shRNA (shGFP) or mouse MKRN1 shRNA (shMKRN1\#1, shMKRN1\#3) were established using the manufacturer's instructions. HEK293T cells were transfected with pLKO.1-shGFP or shMKRN1\#1, \#3, and lentiviral packaging vectors. At $48 \mathrm{~h}$ post transfection, viral supernatants were harvested, filtrated, and added to $3 \mathrm{~T} 3-\mathrm{L} 1$ or $\mathrm{C} 3 \mathrm{H} 10 \mathrm{~T} 1 / 2$ cells. Transduced cells were selected by puromycin treatment for 7 days. MKRN1 overexpression cell lines were produced using pBABE-puro-MKRN1 and pBABE-puro-MKRN1 H307E mutant plasmids, and the packaging cell line Phoenix $E$, as previously described. ${ }^{34}$

In vitro ubiquitination assay. FLAG-MKRN1 was transiently expressed in HEK293T cells, purified by immunoprecipitation using anti-FLAG M2 affinity gel (Sigma-Aldrich, A2220), and eluted by adding FLAG peptide (Sigma-Aldrich, F3290) according to the manufacturer's instructions. Bacterially expressed recombinant GST-PPAR2 was incubated with $200 \mathrm{ng}$ E1 (UBE1, E-305, Boston Biochem, Cambridge, MA, USA), 500 ng E2 (UbcH5c, Boston Biochem, E2-627), $10 \mu \mathrm{g}$ Ubiquitin (Sigma-Aldrich, U6235), 2 mM ATP (Fermentas, Vilnius, Lithuania, R0441) in the absence and presence of FLAG-MKRN1 wild type or H307E mutant in $50 \mu$ l of reaction buffer ( $40 \mathrm{mM}$ Tris- $\mathrm{HCl}, \mathrm{pH} 7.6,50 \mathrm{mM} \mathrm{NaCl}$, and $1 \mathrm{mM} \mathrm{DTT}$ ) for $3 \mathrm{~h}$ at $37^{\circ} \mathrm{C}$. After incubation, reactions were boiled in $1 \%$ SDS to disrupt any protein interactions, diluted with immunoprecipitation buffer to $0.1 \%$ SDS, and immunoprecipitated using $\alpha$-PPAR $\gamma$ mouse monoclonal antibodies (Sigma-Aldrich, $7273 X$ ), followed by western blotting using $\alpha-U b$-HRP or $\alpha$-PPAR $\gamma$ mouse antibodies (Sigma-Aldrich, 7273X). Reactions were directly subjected to western blotting using $\alpha$-MKRN1 antibodies to see MKRN1 self-ubiquitination as a control.

RT-PCR analysis. RNAs were extracted using Trizol (Invitrogen) according to the manufacturer's directions. cDNA was synthesized from total RNA using Reverse Transcriptase M-MLV (Takara Bio), amplified, and then analyzed using the QuantiTect SYBR Green PCR Kit and real-time PCR (Qiagen) with the following primers: human and mouse MKRN1; $5^{\prime}$-GAGCAGGTTCAGAGGACTGG$3^{\prime}$ (forward) and $5^{\prime}$-CACTCTCCCACTGCAGCATA-3' (reverse), mouse PPAR $\gamma$; 5'-CCATTCTGGCCCACCAAC-3' (forward), 5'-AATGCGAGTGGTCTTCCATCA-3' (reverse), human PPAR $\gamma ; 5^{\prime}$-TTCAGAAATGCCTTGCAGTG-3' (forward), $5^{\prime}$-CCAA CAGCTTCTCCTTCTCG-3' (reverse), C/EBP $\alpha ; 5^{\prime}$-GCGGGCAAAGCCAAGAA-3' (forward), 5'-GCGTTCCCGCCGTACC-3' (reverse), aP2; 5'-CACCGCAGACGACA GGAAG-3' (forward), 5'-GCACCTGCACCAGGGC-3' (reverse), CD36; 5'-GGCCAA GCTATTGCGACAT-3' (forward), 5'-CAGATCCGAACACAGCGTAGA-3' (reverse), 36B4; 5'-AGATGCAGCAGATCCGCAT-3' (forward), 5'-GTTCTTGCCCATCAGC ACC-3' (reverse), C/EBP $\beta ; 5^{\prime}$-TGATGCAATCCGGATCAA-3' (forward), $5^{\prime}$-CACGT GTGTTGCGTCAGT-3' (reverse), and C/EBP $\delta ; 5^{\prime}$-GAGAACGAGAAGCTGCATCA-3' (forward), 5'-GGCTGGGCAGTTTTTGAA-3' (reverse). Each gene expression level was normalized to that of the $36 B 4$ gene in the same sample, except for those shown in Figure 1a. The qRT-PCR data shown in Figure 1a were normalized by the GAPDH gene.

Immunoprecipitation and ubiquitination assay. HEK293T cells were lysed using lysis buffer $(50 \mathrm{mM}$ Tris- $\mathrm{HCl}(\mathrm{pH} 7.5), 150 \mathrm{mM} \mathrm{NaCl}$, $0.5 \%$ Triton X-100, $1 \mathrm{mM}$ EDTA, and a protease inhibitor cocktail $(2 \mu \mathrm{g} / \mathrm{ml}$ Aprotinin, $1 \mu \mathrm{g} / \mathrm{ml}$ Pepstatin A, $2 \mu \mathrm{g} / \mathrm{ml}$ Leupeptins, $100 \mu \mathrm{g} / \mathrm{ml}$ PMSF (phenylmethanesulfonylfuoride), Sigma-Aldrich). Cell lysates were incubated with antibodies for $2 \mathrm{~h}$, followed by incubation with protein G Sepharose (GE Healthcare, UK) for $2 \mathrm{~h}$. Immunoprecipitates were boiled in sample buffer for $5 \mathrm{~min}$. In the ubiquitination assay using HA-Ub, H1299 or 3T3-L1 cells transfected with 
the indicated plasmids were harvested by PBS containing $10 \mathrm{nM}$ NEM to prevent deubiquitination. Cells were lysed in $1 \%$ SDS by boiling for $10 \mathrm{~min}$, followed by dilution to $0.1 \%$ SDS by adding lysis buffer, protease inhibitors, and NEM. Lysed samples were immunoprecipitated with $\alpha$-PPAR $\gamma$ antibodies (sc-7273x, Santa Cruz Biotechnology), followed by western blotting. Ubiquitination assays using His$\mathrm{Ub}$ were performed using $6 \mathrm{M}$ guanidinium- $\mathrm{HCl}$ buffer $(\mathrm{pH}$ 8) containing $5 \mathrm{mM}$ NEM. His-ubiquitin-conjugated proteins were purified by incubating with $\mathrm{Ni}^{2}$-NTA agarose beads (Qiagen), followed by washing and elution in sample buffer. ${ }^{22}$

Protein purification, in vitro translation, and in vitro binding assays. pGEX-4T-1-PPAR 2 was expressed in Escherichia coli and purified using glutathione-sepharose 4B (GE Healthcare). Bacteria expressing GST- PPAR $\gamma 2$ were lysed using lysis buffer $(25 \mathrm{mM}$ sodium phosphate buffer $(\mathrm{pH} 7), 300 \mathrm{mM} \mathrm{NaCl}, 20 \mathrm{mM} \beta$-mercaptoethanol, and $5 \%$ glycerol and protease inhibitors). GST-PPAR $\gamma 2$ was purified using GST Sepharose beads following the manufacturer's protocol (GE Healthcare). A TNT T7-coupled reticulocyte lysate system (L4610, Promega, Madison, WI, USA) was used to produce HA-MKRN1 protein. Translated HA-MKRN1 was incubated with GST or GST-PPAR $\gamma 2$ for $2 \mathrm{~h}$, and then Sepharose beads were added followed by an 1-h incubation. These complexes were washed and eluted with $10 \mathrm{mM}$ reduced glutathione, followed by western blotting.

Statistical analyses. All statistical analyses were performed using PRISM (GraphPad Software, San Diego, CA, USA) and results are presented as mean \pm S.D. The $P$-values were designated as follows: ${ }^{*} P<0.05$; ${ }^{*} P<0.01$; ${ }^{* * *} P<0.001$.

\section{Conflict of Interest}

The authors declare no conflict of interest.

Acknowledgements. We thank No-Joon Song and Sung-Pil Choi for their help with cell culture and adipocyte differentiation, and Dr. Peter Tontonoz (University of California at Los Angeles) for helpful discussion. This research was supported by a National Research Foundation of Korea (NRF) grant funded by the Korean government (MEST) (2010-0017787), and by the Cooperative Research Program for Agricultural Science \& Technology Development (2012-PJ008462).

1. Rocha VZ, Libby P. Obesity, inflammation, and atherosclerosis. Nat Rev Cardiol 2009; 6 399-409.

2. Ouchi N, Parker JL, Lugus JJ, Walsh K. Adipokines in inflammation and metabolic disease. Nat Rev Immunol 2011; 11: 85-97.

3. Buechler $\mathrm{C}$, Wanninger J, Neumeier M. Adiponectin, a key adipokine in obesity related liver diseases. World J Gastroenterol 2011; 17: 2801-2811.

4. Park KW, Halperin DS, Tontonoz P. Before they were fat: adipocyte progenitors Cell Metab 2008; 8: 454-457.

5. Cristancho AG, Lazar MA. Forming functional fat: a growing understanding of adipocyte differentiation. Nat Rev Mol Cell Biol 2011; 12: 722-734.

6. Akune T, Ohba S, Kamekura S, Yamaguchi M, Chung UI, Kubota N et al. PPARgamma insufficiency enhances osteogenesis through osteoblast formation from bone marrow progenitors. J Clin Invest 2004; 113: 846-855.

7. Farmer SR. Transcriptional control of adipocyte formation. Cell Metab 2006; 4: 263-273.

8. Tontonoz P, Spiegelman BM. Fat and beyond: the diverse biology of PPARgamma. Annu Rev Biochem 2008; 77: 289-312.

9. Gesta S, Tseng YH, Kahn CR. Developmental origin of fat: tracking obesity to its source. Cell 2007; 131: 242-256.

10. Eguchi J, Yan QW, Schones DE, Kamal M, Hsu CH, Zhang MQ et al. Interferon regulatory factors are transcriptional regulators of adipogenesis. Cell Metab 2008; 7: 86-94.

11. Banerjee SS, Feinberg MW, Watanabe M, Gray S, Haspel RL, Denkinger DJ et al. The Kruppel-like factor KLF2 inhibits peroxisome proliferator-activated receptor-gamma expression and adipogenesis. J Biol Chem 2003; 278: 2581-2584.

12. Hu Y, Davies GE. Berberine increases expression of GATA-2 and GATA-3 during inhibition of adipocyte differentiation. Phytomedicine 2009; 16: 864-873.

13. Tong $\mathrm{Q}$, Dalgin $\mathrm{G}, \mathrm{Xu} \mathrm{H}$, Ting $\mathrm{CN}$, Leiden JM, Hotamisligil GS. Function of GATA transcription factors in preadipocyte-adipocyte transition. Science 2000; 290: 134-138.

14. van Beekum O, Fleskens V, Kalkhoven E. Posttranslational modifications of PPAR-gamma fine-tuning the metabolic master regulator. Obesity (Silver Spring) 2009; 17: 213-219.
15. Adams M, Reginato MJ, Shao DL, Lazar MA, Chatterjee VK. Transcriptional activation by peroxisome proliferator-activated receptor gamma is inhibited by phosphorylation at a consensus mitogen-activated protein kinase site. J Biol Chem 1997; 272: $5128-5132$

16. Pascual G, Fong AL, Ogawa S, Gamliel A, Li AC, Perissi V et al. A SUMOylationdependent pathway mediates transrepression of inflammatory response genes by PPARgamma. Nature 2005; 437: 759-763.

17. Hauser S, Adelmant G, Sarraf P, Wright HM, Mueller E, Spiegelman BM. Degradation of the peroxisome proliferator-activated receptor gamma is linked to ligand-dependent activation. Journal of Biological Chemistry 2000; 275: 18527-18533.

18. Floyd ZE, Stephens JM. Interferon-gamma-mediated activation and ubiquitin-proteasomedependent degradation of PPARgamma in adipocytes. J Biol Chem 2002; 277: 4062-4068.

19. Kilroy G, Kirk-Ballard H, Carter LE, Floyd ZE. The ubiquitin ligase Siah2 regulates PPARgamma activity in adipocytes. Endocrinology 2012; 153: 1206-1218.

20. Han L, Wang P, Zhao G, Wang H, Wang M, Chen J et al. Upregulation of SIRT1 by 17beta-estradiol depends on ubiquitin-proteasome degradation of PPAR-gamma mediated by NEDD4-1. Protein Cell 2013; 4: 310-321.

21. Kim JH, Park SM, Kang MR, Oh SY, Lee TH, Muller MT et al. Ubiquitin ligase MKRN1 modulates telomere length homeostasis through a proteolysis of hTERT. Genes Dev 2005; 19: $776-781$

22. Lee EW, Lee MS, Camus S, Ghim J, Yang MR, Oh W et al. Differential regulation of p53 and p21 by MKRN1 E3 ligase controls cell cycle arrest and apoptosis. EMBO J 2009; 28 : 2100-2113

23. Ko A, Lee EW, Yeh JY, Yang MR, Oh W, Moon JS et al. MKRN1 induces degradation of West Nile Virus capsid protein by functioning as an E3 ligase. $J$ Virol 2010; 84: 426-436.

24. Lee EW, Kim JH, Ahn YH, Seo J, Ko A, Jeong M et al. Ubiquitination and degradation of the FADD adaptor protein regulate death receptor-mediated apoptosis and necroptosis. Nat Commun 2012; 3: 978

25. Ko A, Shin JY, Seo J, Lee KD, Lee EW, Lee MS et al. Acceleration of gastric tumorigenesis through MKRN1-mediated posttranslational regulation of p14ARF. J Natl Cancer Insti 2012; 104: 1660-1672.

26. Omwancha J, Zhou XF, Chen SY, Baslan T, Fisher CJ, Zheng Z et al. Makorin RING finger protein 1 (MKRN1) has negative and positive effects on RNA polymerase II-dependent transcription. Endocrine 2006; 29: 363-373.

27. Tontonoz P, Hu E, Graves RA, Budavari Al, Spiegelman BM. Mppar-Gamma-2 - tissuespecific regulator of an adipocyte enhancer. Genes Dev 1994; 8: 1224-1234.

28. Mueller E, Smith M, Sarraf P, Kroll T, Aiyer A, Kaufman DS et al. Effects of ligand activation of peroxisome proliferator-activated receptor gamma in human prostate cancer. Proc Natl Acad Sci USA 2000; 97: 10990-10995.

29. Agostini M, Schoenmakers E, Mitchell C, Szatmari I, Savage D, Smith A et al. Non-DNA binding, dominant-negative, human PPAR gamma mutations cause lipodystrophic insulin resistance. Cell Metab 2006; 4: 303-311.

30. Iwamoto F, Umemoto T, Motojima K, Fujiki Y. Nuclear transport of peroxisome-proliferator activated receptor \&alpha. J Biochem 2011; 149: 311-319.

31. Umemoto T, Fujiki Y. Ligand-dependent nucleo-cytoplasmic shuttling of peroxisome proliferator-activated receptors, PPARalpha and PPARgamma. Genes Cells 2012; 17: 576-596.

32. Cutress ML, Whitaker HC, Mills IG, Stewart M, Neal DE. Structural basis for the nuclear import of the human androgen receptor. J Cell Sci 2008; 121: 957-968.

33. Wu Z, Bucher NL, Farmer SR. Induction of peroxisome proliferator-activated receptor gamma during the conversion of 3T3 fibroblasts into adipocytes is mediated by C/EBPbeta, C/EBPdelta, and glucocorticoids. Mol Cell Biol 1996; 16: 4128-4136.

34. Park KW, Waki H, Choi SP, Park KM, Tontonoz P. The small molecule phenamil is a modulator of adipocyte differentiation and PPARgamma expression. J Lipid Res 2010; 51: 2775-2784.

55. Lehrke M, Lazar MA. The many faces of PPARgamma. Cell 2005; 123: 993-999.

36. Charo IF. Macrophage polarization and insulin resistance: PPARgamma in control. Cell Metab 2007; 6: 96-98.

37. Tsai YS, Xu L, Smithies O, Maeda N. Genetic variations in peroxisome proliferatoractivated receptor gamma expression affect blood pressure. Proc Natl Acad Sci USA 2009; 106: 19084-19089.

38. Das SK, Chakrabarti R. Role of PPAR in cardiovascular diseases. Recent Patents Cardiovasc Drug Discov 2006; 1: 193-209.

39. Camus S, Higgins M, Lane DP, Lain S. Differences in the ubiquitination of p53 by Mdm2 and the HPV protein E6. FEBS Lett 2003; 536: 220-224.

40. Yang MR, Lee SR, Oh W, Lee EW, Yeh JY, Nah JJ et al. West Nile virus capsid protein induces p53-mediated apoptosis via the sequestration of HDM2 to the nucleolus. Cell Microbiol 2008; 10: 165-176.

41. Zhang JW, Tang QQ, Vinson C, Lane MD. Dominant-negative C/EBP disrupts mitotic clonal expansion and differentiation of 3T3-L1 preadipocytes. Proc Natl Acad Sci USA 2004; 101: 43-47. 